\title{
Altered expression of genes involved in lipid metabolism in obese subjects with unfavourable phenotype
}

\author{
V. H. Telle-Hansen • B. Halvorsen • \\ K. T. Dalen - I. Narverud • N. Wesseltoft-Rao • \\ L. Granlund $\cdot$ S. M. Ulven $\cdot$ K. B. Holven
}

Received: 5 September 2012/ Accepted: 19 December 2012/Published online: 8 January 2013

(C) Springer-Verlag Berlin Heidelberg 2013

\begin{abstract}
Obesity (BMI $\geq 30 \mathrm{~kg} / \mathrm{m}^{2}$ ) increases the risk of developing lifestyle-related diseases. A subgroup of obese individuals has been described as "metabolically healthy, but obese" (MHO). In contrast to at-risk obese (ARO), the MHO phenotype is defined by a favourable lipid profile and a normal or only slightly affected insulin sensitivity, despite the same amount of body fat. The objective was to characterize the metabolic phenotype of MHO subjects. We screened a variety of genes involved in lipid metabolism and inflammation in peripheral blood mononuclear cells (PBMC). Obese subjects (men and women; 18-70 years) with $\mathrm{BMI} \geq 30 \mathrm{~kg} / \mathrm{m}^{2}$ were characterized as $\mathrm{MHO}$ $(n=9)$ or as ARO $(n=10)$. In addition, eleven healthy, normal weight subjects characterized as healthy by the
\end{abstract}

Electronic supplementary material The online version of this article (doi:10.1007/s12263-012-0329-z) contains supplementary material, which is available to authorized users.

V. H. Telle-Hansen · I. Narverud · N. Wesseltoft-Rao ·

S. M. Ulven

Faculty of Health Sciences, Institute of Health, Nutrition and

Management, Oslo and Akershus University College of Applied

Sciences, Postbox 4, St.Olavsplass, 0130 Oslo, Norway

V. H. Telle-Hansen - K. T. Dalen · I. Narverud .

N. Wesseltoft-Rao $\cdot$ K. B. Holven $(\bowtie)$

Department of Nutrition, Institute of Basic Medical Sciences,

Faculty of Medicine, University of Oslo, Postbox 1046,

Blindern, 0317 Oslo, Norway

e-mail: kirsten.holven@medisin.uio.no

B. Halvorsen

Faculty of Medicine, Research Institute for Internal Medicine, Oslo University Hospital Rikshospitalet, University of Oslo,

Postbox 4950, Nydalen, 0424 Oslo, Norway

L. Granlund

Mills DA, Postbox 4644, Sofienberg, 0506 Oslo, Norway same criteria as described for the MHO subjects were included. We found that with similar weight, total fat mass and fat mass distribution, the ARO subjects have increased plasma levels of gamma-glutamyl transpeptidase and free fatty acids. This group also has altered expression levels of a number of genes linked to lipid metabolism in PBMC with reduced gene expression levels of uncoupling protein 2 , hormone-sensitive lipase and peroxisome proliferatoractivated receptor $\delta$ compared with MHO subjects. The present metabolic differences between subgroups of obese subjects may contribute to explain some of the underlying mechanisms causing the increased risk of disease among ARO subjects compared with MHO subjects.

Keywords Lipid metabolism - PBMC $\cdot$ Metabolically healthy obese $\cdot$ Gene expression

$\begin{array}{ll}\text { Abbreviations } \\ \text { ALAT } & \text { Alanine aminotransferase } \\ \text { ALP } & \text { Alkaline phosphatase } \\ \text { ASAT } & \text { Aspartate aminotransferase } \\ \text { BIA } & \text { Bioelectric impedance analysis } \\ \text { CVD } & \text { Cardiovascular disease } \\ \text { FFA } & \text { Free fatty acids } \\ \gamma \text { GT } & \text { Gamma-glutamyl transpeptidase } \\ \text { HOMA } & \text { Homoeostasis model assessment for insulin } \\ & \text { resistance } \\ \text { hsCRP } & \text { High-sensitivity C-reactive protein } \\ \text { HSL } & \text { Hormone-sensitive lipase } \\ \text { MHO } & \text { Metabolically healthy but obese } \\ \text { NAFLD } & \text { Non-alcoholic fatty liver disease } \\ \text { PBMC } & \text { Peripheral blood mononuclear cells } \\ \text { PPAR } \delta & \text { Peroxisome proliferator-activated receptor } \\ & \text { delta } \\ \text { TAG } & \text { Triacylglycerol }\end{array}$


T2DM Type 2 diabetes mellitus

UCP2 Uncoupling protein 2

RT-qPCR Reverse transcription real-time polymerase chain reaction

\section{Introduction}

A subgroup of obese individuals has been described as "metabolically healthy, but obese" (MHO) (Karelis et al. 2004a, b; Sims 2001). In contrast to at-risk obese (ARO) subjects, the MHO phenotype is defined by a favourable lipid profile and a normal to high level of insulin sensitivity, despite the same amount of body fat (Karelis et al. 2004a, b; Wildman 2009). Whether the expression of genes involved in lipid metabolism including uptake, oxidation and de novo lipogenesis differs between MHO and ARO subjects remains to be elucidated. To study human hepatic gene expression in vivo is challenging. Peripheral blood mononuclear cells (PBMC) include monocytes and lymphocytes and are easily obtainable in humans. Expression pattern of genes involved in lipid metabolism is reflected in human PBMC (Bouwens et al. 2007, 2008, 2010; Marx et al. 2001, 2002). Additionally, since liver, adipose tissue and PBMC evolutionary derive from the same body compartment (Hotamisligil 2006), PBMC may be a good model system for reflecting important metabolic changes in the liver. In order to understand the underlying molecular mechanisms determining why a subgroup of obese subjects manages to keep a healthy metabolic profile compared with other obese subjects with similar BMI, one approach would be to study the gene expression in PBMC. The aim of the present study was therefore to characterize the metabolic phenotype of MHO subjects compared with ARO subjects by screening a variety of genes involved in lipid metabolism in PBMC.

\section{Methods}

\section{Study population}

Obese subjects (men and women; 18-70 years) with BMI $\geq 30 \mathrm{~kg} / \mathrm{m}^{2}$ were included in this study and were characterized as $\mathrm{MHO}(n=9)$ when three out of the following five criteria were fulfilled $\left(\mathrm{HOMA}_{\text {ir }}\right.$ index $\leq 1.95$; triacylglycerol (TAG) $\leq 1.7 \mathrm{mmol} / \mathrm{L}$; total cholesterol $\leq 5.2 \mathrm{mmol} / \mathrm{L} ; \mathrm{LDL}$ cholesterol $\leq 2.6 \mathrm{mmol} / \mathrm{L}$ and $\mathrm{HDL}$ cholesterol $\geq 1.3 \mathrm{mmol} / \mathrm{L}$ ) or as ARO subjects $(n=10)$ when four out of the following five criteria were fulfilled $\left(\mathrm{HOMA}_{\mathrm{ir}}\right.$ index $\geq 1.95$; TAG $\geq 1.7 \mathrm{mmol} / \mathrm{L} ;$ total cholesterol $\geq 5.2 \mathrm{mmol} / \mathrm{L}$; LDL cholesterol $\geq 2.6 \mathrm{mmol} / \mathrm{L}$ and $\mathrm{HDL} \leq 1.3 \mathrm{mmol} / \mathrm{L}$ ).
The criteria used in the present study are based on the National Cholesterol Education Program's Adult Treatment Panel III report (ATP III) for lipid profiles as previously described by Karelis et al. (2004a, b). Furthermore, eleven healthy, normal weight subjects characterized as healthy when four out of five of the same criteria described for the MHO subjects were included. Exclusion criteria were T2DM; kidney, liver, gall bladder, coronary, endocrine or chronic rheumatic disease; malign cancer the last 5 years; hypertension ( $\geq 160 / 100)$; pregnancy and lactation. Regular use of anti-inflammatory, lipid-lowering and antihypertensive medications was not permitted. The study was approved by the Regional Committee of Medical Ethics (approval no. 6.2008.1368) and by the Norwegian Social Science Data Services (approval no. 19667). Written informed consent for participation was obtained from each participant, and the study complied with the Declaration of Helsinki.

Measurements of body composition

Subjects wore light clothing and no shoes. Two trained persons performed all measurements. Height was measured by a wall-mounted stadiometer to the nearest $0.1 \mathrm{~cm}$. Weight (to the nearest $0.1 \mathrm{~kg}$ ), BMI and body composition were estimated by the Tanita BC-418 bioelectric impedance analysis (BIA), a hand-to-foot system, according to the manufacturer's manual (Tanita Corporation, Tokyo, Japan). One kilogram was subtracted from the measured value to correct for light clothing. Waist circumference and hip circumference were measured to the nearest millimetre with a standard, non-stretch tape. Waist circumference was measured at the point midway between the iliac crest and the lower rib margin, while hip circumference was measured at the maximum circumference of the buttocks posterior and the symphysis anterior. All subjects were measured while standing in a relaxed position and with normal respiration. Age, height and body type (all were routinely classified as "standard" body type) were entered before measuring. BIA measurements were carried out at a frequency of $50 \mathrm{kHz}$.

Blood sampling

The day prior to blood sampling the subjects were told to refrain from alcohol consumption and vigorous physical activity. Venous blood samples were drawn after an overnight fast $(\geq 12 \mathrm{~h})$. Serum was obtained from silica gel tubes [Becton-Dickinson (BD) vacutainer] and kept at room temperature for at least $30 \mathrm{~min}$, until centrifugation $(1,500 \mathrm{~g}, 12 \mathrm{~min})$. Serum was kept at room temperature and immediately prepared for subsequent analysis of routine laboratory analyses or aliquoted and stored at $-80{ }^{\circ} \mathrm{C}$ until further analyses. Plasma was obtained from EDTA tubes 
(BD vacutainer), immediately placed on ice and centrifuged within $10 \mathrm{~min}\left(1500 \mathrm{~g}, 4{ }^{\circ} \mathrm{C}, 10 \mathrm{~min}\right)$. Plasma samples were aliquoted and stored at $-80{ }^{\circ} \mathrm{C}$ until further analyses.

\section{Routine laboratory analysis}

Fasting serum high-sensitivity C-reactive protein (hsCRP), total cholesterol, LDL cholesterol, HDL cholesterol, TAG, glucose, C-peptide, insulin, HbA1c, gamma-glutamyl transpeptidase $(\gamma \mathrm{GT})$, alkaline phosphatase (ALP), alanine aminotransferase (ALAT) and aspartate aminotransferase (ASAT) were measured by standard methods at a routine laboratory (Fürst Medical Laboratory, Norway). Free fatty acids (FFA) were measured by standard methods at Oslo University Hospital, Norway.

PBMC and RNA isolation and RT-qPCR analysis

After blood collection, PBMC were isolated using BD Vacutainer Cell Preparation tubes according to the manufacturer's instructions (Becton, Dickinson and Company, NJ 07417, USA). Pellets were frozen and stored at $-80{ }^{\circ} \mathrm{C}$ prior to RNA isolation.

Total RNA was isolated from all PBMC samples using RNeasy mini kit (Qiagen, Hilden, Germany) with lysis buffer-added $\beta$-mercaptoethanol according to the manufacturer's instructions and stored at $-80{ }^{\circ} \mathrm{C}$. RNA quantity and quality measurements were performed using ND 1000 Spectrophotometer (Saveen Werner, Malmö, Sweden) and Agilent Bioanalyser (Agilent Technologies, Santa Clara, CA, USA), respectively. All samples had RNA integrity number $(\mathrm{RIN})>8$. Four hundred nanogram of RNA was reverse transcribed by high-capacity RNA-to-cDNA kit (cat\# 4387406, Applied Biosystems, Foster City, CA, USA). Reverse transcription real-time polymerase chain reaction (RT-qPCR) was performed on an ABI PRISM $7900 \mathrm{HT}$ or $7500 \mathrm{HT}$ (single assays) Sequence Detector System (Applied Biosystems). Custom-designed TaqMan Low-Density array (LDA) cards (Applied Biosystems) and Taqman or Power SYBR green (designed in Primer Express software version 1) (Applied Biosystems) single assays were used for RT-qPCR amplification of the target genes, all shown in the supplementary table. Due to errors in five of the wells on the LDA card, ATP-binding cassette (ABC) A1, ABCG1, carnitine palmitoyltransferase 1A (CPT1A), CD36 molecule (CD36) and acyl-coenzyme A oxidase 1 (ACOX1) were not included in the analysis. TATA-binding protein (TBP) was selected as housekeeping gene based on running a TaqMan Human Endogenous Control Plate-test (data not shown). Ten microlitre of
cDNA (200 ng) was added to water and Taqman master mix to a total volume of $100 \mu$ before applying to the LDA card and centrifuged according to the manufacturer's protocol. For the single assays, cDNA was diluted 1:5. The relative mRNA level for each transcript was calculated by the $\Delta \Delta$ cycle threshold $(\mathrm{Ct})$ method (Livak and Schmittgen 2001). Briefly, the $\mathrm{Ct}$ values of each target gene were subtracted from the $\mathrm{Ct}$ values of the housekeeping gene TBP $(=\Delta \mathrm{Ct})$. Target gene $\Delta \Delta \mathrm{Ct}$ of MHO or ARO individuals was calculated as $\Delta \mathrm{Ct}$ minus median $\Delta \mathrm{Ct}$ of normal weight individuals. The fold change in mRNA expression was calculated as $2^{-\Delta \Delta \mathrm{Ct}}$.

Enzyme immunoassay (EIA)

Serum fetuin-A, adiponectin, CD40 ligand (CD40L), leptin, resistin and IL-6 were measured by EIA from R\&D Systems (Minneapolis, MN, USA) according the manufacturer's instructions.

\section{Statistics}

As the number of subjects in the study was relatively small, the significance was assessed by nonparametric analyses and correction for multiple testing was performed with Kruskal-Wallis test, followed by Mann-Whitney $U$ test at significant values. All values are presented as median (25-75 percentiles). All analyses were performed using SPSS for Windows (version 19.0). Probability values (asymptotic) were considered statistically significant at a value of $\leq 0.05$.

\section{Results}

Characteristics of the participants

The age of the subjects were 49 years (42-63 years; MHO $[n=9]), 52$ years $(43-59$ years; ARO $[n=10])$ and 47 years (42-54 years; normal weight $[n=11])$ with a BMI of $33\left(30-37 \mathrm{~kg} / \mathrm{m}^{2}\right), 32\left(30-34 \mathrm{~kg} / \mathrm{m}^{2}\right)$ and 23 $\left(21-24 \mathrm{~kg} / \mathrm{m}^{2}\right)$, respectively. There were no significant differences between the two obese groups in weight or BMI (Table 1). As expected, the parameters included in the inclusion criteria were significantly different between the ARO subjects compared with the MHO subjects (Table 1). hsCRP was significantly higher in both MHO subjects $(P=0.033)$ and ARO subjects $(P=0.020)$ compared with healthy, normal weight subjects. There was no significant difference in the level of hsCRP between the two obese groups. 
Table 1 Characteristics of the population (median with $25-75$ percentiles)

${ }^{a}$ Normal weight versus at-risk obese (ARO)

${ }^{\mathrm{b}}$ Normal weight versus metabolically healthy but obese (MHO)

c ARO versus MHO

${ }^{\mathrm{d}}$ Homoeostasis model assessment for insulin resistance: (fasting plasma insulin $(\mathrm{mU} / \mathrm{L}) \times$ fasting plasma glucose $(\mathrm{mmol} / \mathrm{L})) / 22.5$

$(1 \mathrm{mU} / \mathrm{L}=6.945 \mathrm{pmol} / \mathrm{L})$

\begin{tabular}{lllllll}
\hline & $\begin{array}{l}\text { Normal weight } \\
n=11\end{array}$ & $\begin{array}{l}\text { ARO } \\
n=10\end{array}$ & $\begin{array}{l}\text { MHO } \\
n=9\end{array}$ & $P^{\mathrm{a}}$ & $P^{\mathrm{b}}$ & $P^{\mathrm{c}}$ \\
\hline Gender (m/f) & $4 / 7$ & $9 / 1$ & $5 / 4$ & & & \\
Age (years) & $47(42-54)$ & $52(43-59)$ & $49(42-63)$ & - & - & - \\
Weight $(\mathrm{kg})$ & $65.8(63.1-79.1)$ & $102.7(96.0-113.8)$ & $104.4(93.3-110.6)$ & $<0.001$ & $<0.001$ & - \\
BMI $\left(\mathrm{kg} / \mathrm{m}^{2}\right)$ & $23(21-24)$ & $32(30-34)$ & $33(30-37)$ & $<0.001$ & $<0.001$ & - \\
TAG $(\mathrm{mmol} / \mathrm{L})$ & $0.6(0.4-0.9)$ & $2.3(1.7-2.7)$ & $1.0(0.8-1.3)$ & $<0.001$ & $<0.001$ & $<0.001$ \\
Total cholesterol & $4.7(4.2-5.0)$ & $5.8(5.4-6.1)$ & $5.1(4.3-5.2)$ & $<0.001$ & - & 0.002 \\
$\quad(\mathrm{mmol} / \mathrm{L})$ & & & & & & \\
HDL $(\mathrm{mmol} / \mathrm{L})$ & $1.6(1.4-2.2)$ & $1.1(0.9-1.1)$ & $1.4(1.2-1.5)$ & $<0.001$ & 0.032 & 0.001 \\
LDL (mmol/L) & $2.3(2.1-2.6)$ & $3.8(3.4-3.9)$ & $2.9(2.6-3.3)$ & $<0.001$ & 0.009 & 0.002 \\
Glucose (mmol/L) & $5.1(4.3-5.3)$ & $5.6(4.1-6.3)$ & $5.4(4.8-5.7)$ & 0.044 & - & - \\
Insulin (pmol/L) & $40(19-59)$ & $75(60-107)$ & $65(52-102)$ & 0.001 & 0.012 & 0.033 \\
HOMA & $1.4(0.6-2.0)$ & $3.0(2.3-4.3)$ & $2.2(1.7-3.7)$ & $<0.001$ & 0.015 & 0.009 \\
HbA1c $(\%)$ & $5.1(4.9-5.4)$ & $5.3(5.0-5.9)$ & $5.6(5.3-5.7)$ & - & 0.039 & - \\
C-peptide (pmol/L) & $482(279-584)$ & $942(821-1171)$ & $889(754-1155)$ & $<0.001$ & $<0.001$ & - \\
hsCRP (mg/L) & $0.5(0.3-1.1)$ & $1.6(0.8-3.5)$ & $2.2(0.8-2.7)$ & 0.020 & 0.033 & - \\
\hline
\end{tabular}

Anthropometric measurements and adipokines in serum

Total fat mass, trunk fat mass, fat-free mass, hip and waist circumference were measured, and waist/hip ratio was calculated in all subjects (Fig. 1). Anthropometric measures concerning fat distribution did not differ between the two obese groups. Both MHO and ARO subjects had larger total fat mass, trunk fat mass, hip circumference, waist circumference, waist/hip ratio $(P<0.001$ for all parameters for both groups) and fat-free mass $(P=0.053$ and $P=0.003$ ) than normal weight subjects. In addition, the circulating levels of leptin, adiponectin and resistin were measured. There was no difference in any of these parameters between the two obese groups. As expected, normal weight subjects had lower levels of leptin compared with both obese groups, while adiponectin levels were higher only compared with ARO subjects (Fig. 2).

Circulating markers of hepatic fat accumulation and inflammation

We measured established markers of liver dysfunction and liver fat accumulation such as $\gamma \mathrm{GT}$, ASAT, ALAT, ALP as well as fetuin-A. $\gamma$ GT levels were significantly higher in ARO subjects compared with MHO $(P=0.008)$ and normal weight subjects $(P<0.001)$. In addition, MHO subjects had increased $\gamma \mathrm{GT}$ concentration levels compared with normal weight subjects $(P=0.043)$ (Fig. 3). There were no significant differences in ALAT, ASAT, ALP or fetuin-A between any of the groups (Fig. 3). We also analysed the circulating level of the inflammatory markers
IL6 and CD40L. There was no significant difference between the two obese groups; however, both obese groups had significantly elevated serum levels of IL6 and CD40L compared with normal weight subjects (data not shown).

Free fatty acids in plasma

The availability of FFA from circulation may influence the progression of fat accumulation in the liver (MendezSanchez et al. 2007). ARO subjects had significant higher plasma levels of FFA $(P=0.020)$ compared with $\mathrm{MHO}$ subjects (Fig. 4).

\section{PBMC gene expression}

The increase in plasma FFA and $\gamma \mathrm{GT}$ in ARO subjects may point to development of fat accumulation and lipid disturbances in the liver. Expression and regulation of genes involved in lipid metabolism in PBMC have previously been shown to reflect hepatic changes (Bouwens et al. 2007, 2008). The expression levels of selected genes involved in lipid uptake, transport, lipolysis, de novo lipogenesis and fatty acid oxidation were analysed (Table 2). Additionally, we analysed the expression of the transcription factors PPAR $\delta$ and LXR $\alpha$ which have been described as fatty acid sensors regulating genes involved in lipid metabolism (Sanderson et al. 2009; Strable and Ntambi 2010). Whereas there were no differences in PBMC gene expression levels of UCP2, HSL and PPAR $\delta$ between normal weight and MHO subjects, ARO subjects had significantly lower expression levels of these genes 

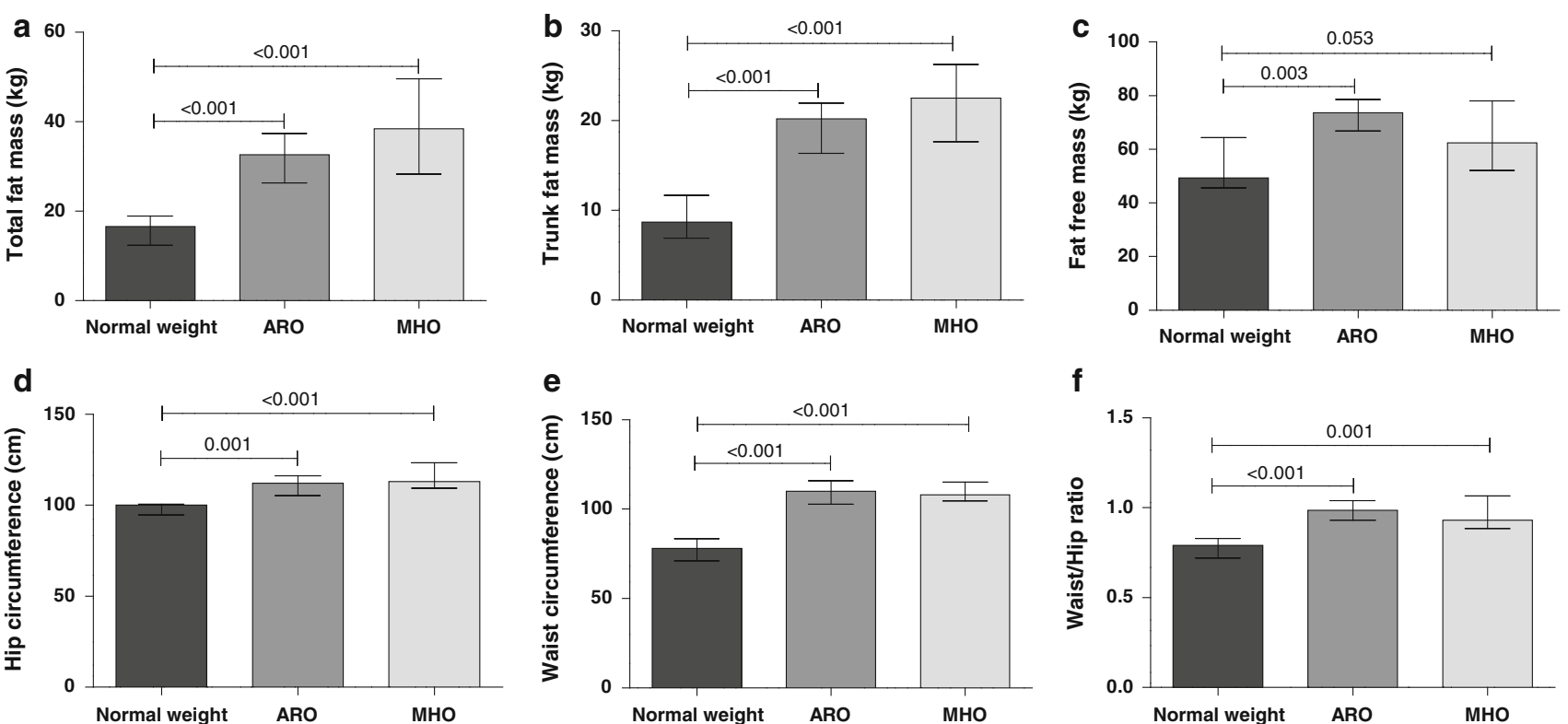

Fig. 1 Anthropometric measurements in normal weight $(n=11)$, ARO $(n=10)$ and MHO subjects $(n=9)$. Total fat mass (a), trunk fat mass (b), fat-free mass (c), hip circumference (d), waist

circumference (e) and waist/hip ratio (f). ARO at-risk obese, $M H O$ metabolically healthy but obese. Data are shown as median, and bars indicate (25-75) percentiles
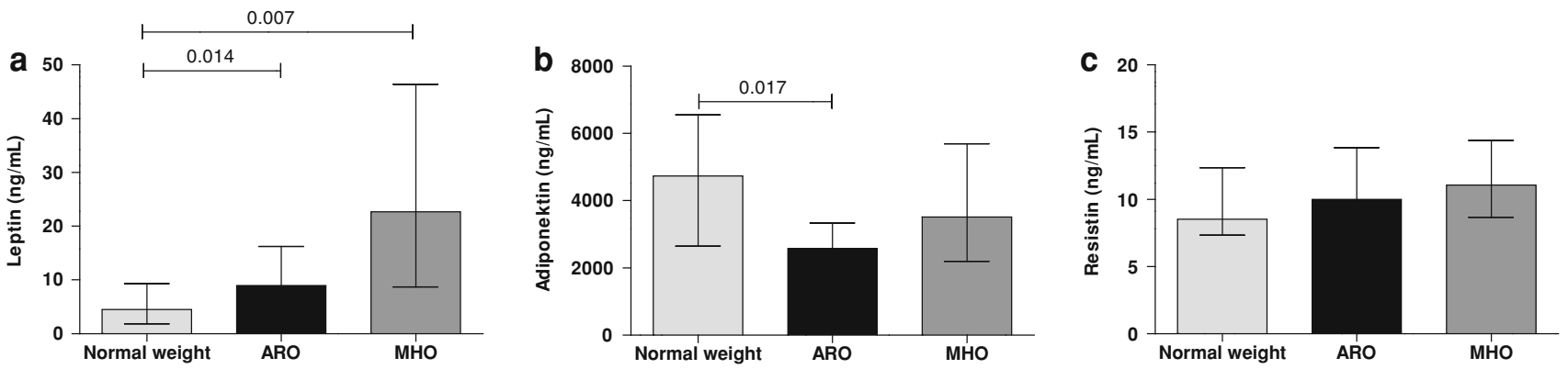

Fig. 2 Plasma levels of leptin (a), adiponectin (b) and resistin (c) in normal weight $(n=11)$, ARO $(n=10)$ and MHO $(n=9)$ subjects. ARO at-risk obese, $M H O$ metabolically healthy but obese. Data are shown as median, and bars indicate (25-75) percentiles

compared with both normal weight subjects $(P=0.041$, $P=0.035$ and $P=0.05$, respectively) and MHO subjects $(P=0.003, \quad P=0.003$ and $P=0.006$, respectively) (Fig. 5). Minor differences between normal weight subjects and the two obese groups were observed for some genes; however, for the majority of genes, we found no differences in the expression level between the groups (Table 2).

\section{Discussion}

In the present study, we have screened a variety of markers associated with fat accumulation in the liver and lipid metabolism in order to characterize differences in metabolic pathways between MHO and ARO subjects. We show that despite similar weight, total fat mass and fat mass distribution, ARO subjects have increased plasma levels of $\gamma \mathrm{GT}$ and FFA, and reduced PBMC gene expression level of UCP2, HSL and PPAR $\delta$ compared with MHO subjects. These metabolic differences may contribute to explain some of the underlying mechanisms causing increased risk of disease among ARO subjects compared with MHO subjects.

MHO subjects are characterized with a favourable lipid profile and normal insulin sensitivity despite an excessive fat mass. The mechanistic explanation as to how a subgroup of obese subjects manages to obtain these metabolically favourable conditions is poorly understood. Elevated levels of circulating $\gamma \mathrm{GT}$ and ALAT, without fatty liver or hepatic dysfunction, in healthy and asymptomatic subjects may predict future development of metabolic disease (Vozarova et al. 2002; Wannamethee et al. 2005). We found that ARO subjects had higher levels of the liver marker $\gamma \mathrm{GT}$ than both MHO and normal weight 

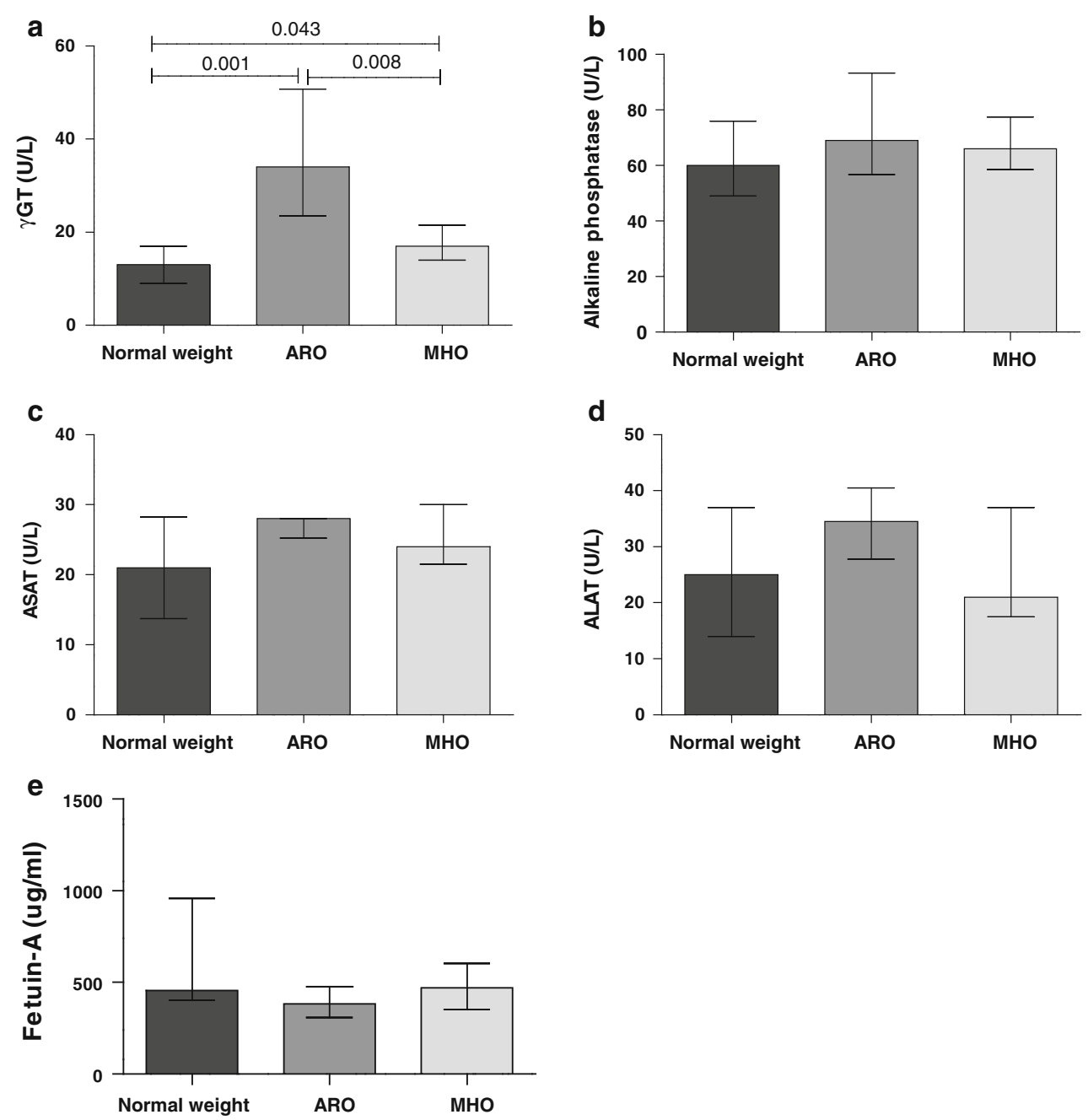

Fig. 3 Plasma levels of $\gamma \mathrm{GT}(\mathbf{a}), \operatorname{ALP}(\mathbf{b})$, ASAT (c), ALAT $(\mathbf{d})$ and fetuin-A $(\mathbf{e})$ in normal weight $(n=11)$, ARO $(n=10)$ and MHO subjects $(n=9)$. ARO at-risk obese, $M H O$ metabolically healthy but obese. Data are shown as median, and bars indicate (25-75) percentiles

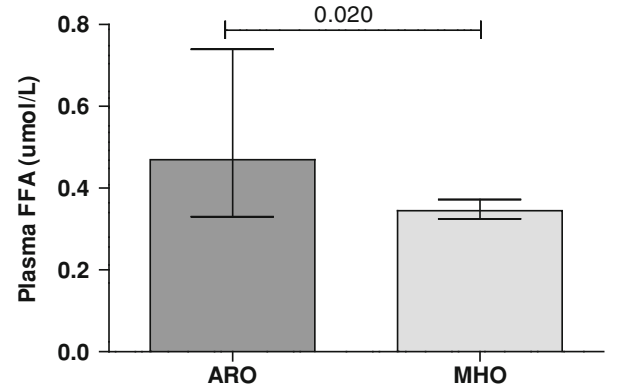

Fig. 4 Plasma levels of FFA in ARO $(n=10)$ and MHO subjects $(n=9)$. ARO at-risk obese, $F F A$ free fatty acids, $M H O$ metabolically healthy but obese. Data are shown as median, and bars indicate (25-75) percentiles

individuals. This is in line with reports from a previous study where the level of ALAT, even within the normal range, was shown to be significantly associated with obesity phenotypes (Mojiminiyi et al. 2010). We were unable to distinguish between subcutaneous and visceral fat; however, there were no differences between MHO and ARO subjects regarding trunk fat mass or waist circumference. Even so, the level of the different fat depots may still differ between these two obese groups as shown by Brochu and co-workers who found that the MHO subjects had up to $49 \%$ less visceral fat mass (Brochu et al. 2001).

In the present study, we found genes related to $\beta$-oxidation to be changed. PBMC gene expression level of UCP2 in ARO subjects was significantly decreased compared with both normal weight and MHO subjects. UCP2 has previously been associated with obesity (Dalgaard 2011; Dalgaard and Pedersen 2001). UCP2 is important for uncoupling respiration and promotes mitochondrial fatty acid oxidation (Fisler and Warden 2006), although the physiological significance of its uncoupling activity is discussed (Pecqueur et al. 2009).

We found that ARO subjects had significant lower expression of PPAR $\delta$ than the MHO subjects. PPAR $\delta$ has 
Table 2 PBMC mRNA expression levels in normal weight, at-risk obese (ARO) and metabolically healthy but obese (MHO) subjects (median with $25-75$ percentiles)

\begin{tabular}{|c|c|c|c|c|c|c|}
\hline $\begin{array}{l}\text { Target gene } \\
\text { Lipids }\end{array}$ & $\begin{array}{l}\text { Normal weight }(n=11) \\
\text { Target gene/TBP }\end{array}$ & $\begin{array}{l}\text { ARO }(n=10) \\
\text { Target gene/TBP }\end{array}$ & $\begin{array}{l}\text { MHO }(n=9) \\
\text { Target gene/TBP }\end{array}$ & $P^{\mathrm{a}}$ & $P^{\mathrm{b}}$ & $P^{\mathrm{c}}$ \\
\hline $\mathrm{ADRP}^{\mathrm{d}}$ & $1.08(0.9-1.2)$ & $1.05(0.9-1.2)$ & $1.14(0.9-1.3)$ & - & - & - \\
\hline $\mathrm{CD} 36^{\mathrm{d}}$ & $0.85(0.8-1.3)$ & $0.96(0.8-1.2)$ & $1.14(0.8-1.4)$ & - & - & - \\
\hline CPT1A ${ }^{d}$ & $1.18(0.9-1.3)$ & $0.86(0.8-1.3)$ & $1.14(0.7-1.6)$ & - & - & - \\
\hline $\mathrm{FABP}^{\mathrm{d}}$ & $0.64(0.4-1.5)$ & $0.46(0.3-0.7)$ & $0.69(0.6-0.9)$ & - & - & - \\
\hline LIPE & $0.38(0.3-0.5)$ & $0.28(0.2-0.4)$ & $0.46(0.4-0.5)$ & 0.035 & - & 0.003 \\
\hline $\mathrm{LPL}^{\mathrm{d}}$ & $0.59(0.3-1.5)$ & $0.76(0.4-1.6)$ & $0.56(0.2-1.9)$ & - & - & - \\
\hline LXR & $0.20(0.2-0.3)$ & $0.22(0.2-0.3)$ & $0.22(0.2-0.3)$ & - & - & - \\
\hline NAMPT & $3.33(3.2-4.3)$ & $3.23(2.8-3.5)$ & $2.87(2.5-3.5)$ & - & - & - \\
\hline PLA2G4A & $0.33(0.2-0.3)$ & $0.25(0.2-0.3)$ & $0.29(0.2-0.4)$ & - & - & - \\
\hline PLA2G7 & $0.55(0.4-1.0)$ & $0.40(0.2-0.6)$ & $0.75(0.3-1.0)$ & - & - & - \\
\hline PLTP & $0.14(0.1-0.2)$ & $0.11(0.1-0.2)$ & $0.11(0.1-0.2)$ & - & - & - \\
\hline PPAR $\delta$ & $1.39(1.2-1.8)$ & $1.08(1.0-1.3)$ & $1.40(1.4-1.6)$ & 0.049 & - & 0.006 \\
\hline $\operatorname{PPAR} \gamma \mathrm{C} 1 \beta$ & $0.49(0.4-0.5)$ & $0.41(0.3-0.4)$ & $0.41(0.4-0.5)$ & - & - & - \\
\hline PTGS2 & $0.26(0.2-0.3)$ & $0.18(0.2-0.2)$ & $0.24(0.2-0.3)$ & 0.009 & - & - \\
\hline TLR2 & $3.05(2.1-3.5)$ & $1.63(1.4-2.8)$ & $2.39(1.6-2.8)$ & - & - & - \\
\hline TLR4 & $3.13(2.4-3.8)$ & $2.43(2.1-3.2)$ & $3.22(2.5-3.9)$ & - & - & - \\
\hline TLR6 & $0.76(0.7-1.1)$ & $0.67(0.5-0.7)$ & $0.63(0.6-0.9)$ & - & - & - \\
\hline SREBP1 & $1.04(0.9-1.4)$ & $0.78(0.7-1.0)$ & $1.09(0.9-1.2)$ & - & - & - \\
\hline SCD1 & $0.29(0.2-0.3)$ & $0.23(0.2-0.2)$ & $0.29(0.2-0.4)$ & - & - & - \\
\hline $\mathrm{UCP} 2$ & 31.77 (28.5-36.3) & $27.38(26.2-30.0)$ & $36.41(31.1-42.2)$ & 0.041 & - & 0.003 \\
\hline \multicolumn{7}{|l|}{ Inflammation } \\
\hline $\mathrm{CD} 40$ & $0.74(0.7-1.1)$ & $0.71(0.6-0.9)$ & $1.05(0.8-1.3)$ & - & - & - \\
\hline CD8A & $8.56(6.3-16.2)$ & $11.82(7.5-17.3)$ & $11.27(9.1-13.9)$ & - & - & - \\
\hline CD3E & $28.84(23.4-33.3)$ & $23.74(22.4-26.2)$ & $31.17(25.0-32.7)$ & - & - & - \\
\hline CD40LG & $1.76(1.1-2.0)$ & $1.62(1.4-1.9)$ & $1.76(1.5-1.9)$ & - & - & - \\
\hline CXCL16 & $1.52(1.4-2.2)$ & $1.41(1.1-1.6)$ & $2.00(1.5-2.2)$ & - & - & - \\
\hline FOXP3 & $0.30(0.2-0.4)$ & $0.25(0.2-0.3)$ & $0.36(0.3-0.4)$ & - & - & - \\
\hline GATA3 & $3.49(3.0-5.0)$ & $3.10(2.8-3.9)$ & $3.80(3.5-4.3)$ & - & - & - \\
\hline $\mathrm{IFN} \gamma$ & $0.16(0.1-0.3)$ & $0.29(0.2-0.5)$ & $0.25(0.2-0.4)$ & - & - & - \\
\hline IL1 $\beta$ & $0.83(0.7-1.5)$ & $0.59(0.5-0.8)$ & $0.65(0.6-1.0)$ & 0.007 & 0.037 & - \\
\hline IL4 & ND & ND & ND & & & \\
\hline IL8 & ND & ND & ND & & & \\
\hline IL18 & $0.26(0.2-0.3)$ & $0.19(0.2-0.3)$ & $0.23(0.2-0.3)$ & - & - & - \\
\hline TBX21 & $4.49(3.7-5.4)$ & $6.32(4.1-7.2)$ & $5.79(4.3-6.2)$ & - & - & - \\
\hline TGFB2 & $0.09(0.1-0.2)$ & $0.12(0.1-0.3)$ & $0.16(0.1-0.2)$ & - & - & - \\
\hline $\mathrm{TNF}$ & $1.52(1.1-1.7)$ & $1.34(1.1-1.6)$ & $1.42(1.2-1.8)$ & - & - & - \\
\hline \multicolumn{7}{|c|}{ Autophagi/ER stress } \\
\hline BAG3 & $0.43(0.3-0.5)$ & $0.33(0.2-0.4)$ & $0.44(0.4-0.5)$ & - & - & - \\
\hline SQSTM1 & $5.50(4.9-6.4)$ & $5.13(4.3-5.3)$ & $6.06(5.0-6.5)$ & - & - & - \\
\hline \multicolumn{7}{|c|}{ Markers associated with obesity } \\
\hline CTSS & $82.53(68.8-123.6)$ & $75.82(64.2-97.6)$ & $100.80(76.3-114.6)$ & - & - & - \\
\hline F3 & ND & ND & ND & & & \\
\hline PLAT & ND & ND & ND & & & \\
\hline TFPI & $0.40(0.3-0.8)$ & $0.60(0.5-0.8)$ & $0.58(0.6-0.9)$ & - & - & - \\
\hline RBP4 & ND & ND & ND & & & \\
\hline SERPINE & $0.07(0.1-0.1)$ & $0.14(0.1-0.2)$ & $0.17(0.1-0.2)$ & - & 0.014 & - \\
\hline
\end{tabular}

${ }^{a}$ Normal weight versus ARO

b Normal weight versus MHO

c ARO versus MHO

d Single assays 

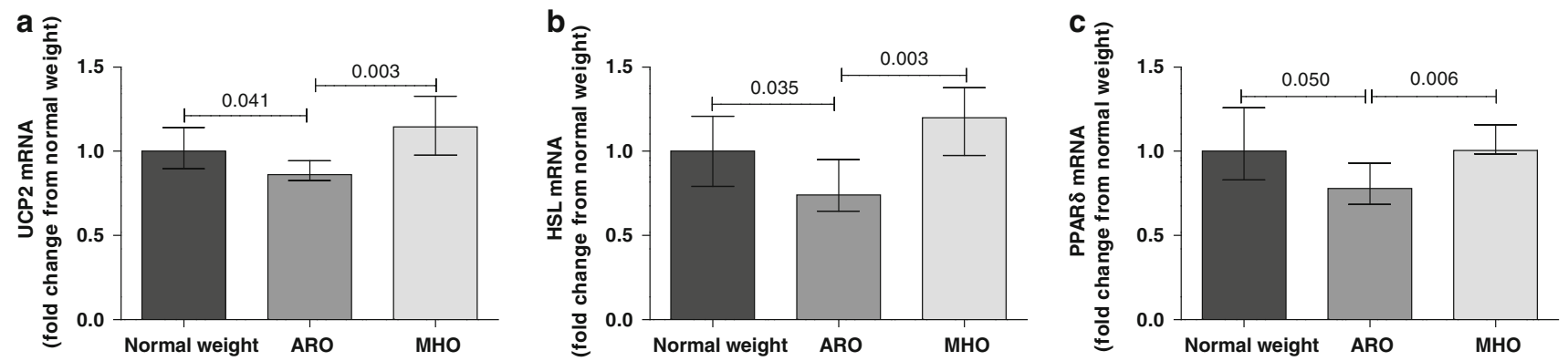

Fig. 5 PBMC mRNA levels of UCP2 (a), HSL (b) and PPAR $\delta(\mathbf{c})$ in normal weight $(n=11)$, ARO $(n=10)$ and MHO subjects $(n=9)$. The figures are shown as fold change (median) from normal weight subjects, and the bars indicate (25-75) percentiles. $A R O$ at-risk obese,
$H S L$ hormone-sensitive lipase, $M H O$ metabolically healthy but obese, $P B M C$ peripheral blood mononuclear cells, PPAR $\delta$ peroxisome proliferator-activated receptor delta, $U C P 2$ uncoupling protein 2 been suggested to be a fatty acid sensor, and recent studies have shown that activation of PPAR $\delta$ may lead to beneficial effects on lipoprotein and glucose metabolism (Ooi et al. 2011). Activation with the synthetic PPAR $\delta$ agonist GW501516 decreases plasma TAG and plasma FFA in dyslipidaemic subjects with central obesity (Ooi et al. 2011). PPAR $\delta$ appears to sense and respond to elevated FFA by up-regulating genes such as Lipin 2 (Sanderson et al. 2009), and in mice models $\operatorname{PPAR} \delta$ agonists have previously been shown to protect against weight gain, steatosis, reduced HDL levels and elevated TAG levels, while $\operatorname{PPAR} \delta$ null mice were more susceptible to weight gain (Barish et al. 2006). This collectively suggests that activation of PPAR $\delta$ protects against obesity and/or dyslipidaemia. One interpretation of our results may be that ARO subjects have an impaired lipid metabolism with a slower fatty acid turnover due to impaired fatty acid oxidation. In the present study, ARO subjects had higher plasma levels of FFA than MHO subjects. If fatty acid oxidation and lipid storage is impaired, one might expect that FFA will remain in plasma, leading to increased levels.

A tight regulation of hepatic TAG synthesis, hydrolysis and $\beta$-oxidation is required to prevent lipid accumulation. HSL was initially observed in adipose tissue, but observations in mice suggest that HSL is also expressed in liver (Mulder et al. 2003; Reid et al. 2008; Sekiya et al. 2008). Hepatic overexpression of HSL (in cells) is shown to reduce hepatic steatosis by promoting $\beta$-oxidation, and it has therefore been suggested that HSL may be a therapeutic target for the treatment of fatty liver in human subjects (Reid et al. 2008).

Our data are in line with the previous observation from Taskinen and co-workers (Taskinen et al. 2011), who showed that hypertriglyceridaemia in obese men with similar BMI and waist circumference was caused by increased hepatic secretion of VLDL (induced by increased liver fat) in combination with severely impaired clearance of triglyceride-rich $\mathrm{VLDL}_{1}$ particles. The liver fat content seemed to be the driving force for the overproduction of VLDL $_{1}$-triglycerides and apoB. In the present study, we extend and support these novel findings by showing reduced $\mathrm{PBMC}$ gene expression, potentially reflecting liver expression, of UCP-2 and PPAR $\delta$ in ARO subjects compared with MHO subjects, further supporting such a notion.

Furthermore, also in line with our data, in a recent study, the specific PPAR $\delta$ agonist GW501516 showed potent hypolipidaemic actions in a two-week randomized controlled study with six healthy moderately overweight subjects in each intervention arm (Riserus et al. 2008). The fasting plasma TAG concentration was reduced by $30 \%$, and there was a concomitantly lowering of the fasting nonesterified fatty acid concentrations which were followed by an increase in the oxidation of fatty acids measured as increased gene expression of CPT1b in skeletal muscle. This mechanism was shown to be unique for the PPAR $\delta$ agonist and not seen with the PPAR $\alpha$ agonist. In the present study, we show that ARO subjects have reduced PBMC gene expression of PPAR $\delta$, increased level of nonesterified fatty acids in circulation and decreased PBMC expression UCP-2, which promotes mitochondrial fatty acid oxidation.

The strength of the study, even though being descriptive in nature, is that we have thorough clinical description, extensive anthropometric measurements, gene expression analysis and plasma markers in the two obese groups. The limitations of the study are the relative small number of subjects in each group, the lack of measurements of FFA in the normal weight subjects and the skewed gender distribution. When recalculating the data, using only the male population in each group, the same pattern was found between MHO and ARO subjects and the level of significance was maintained for most of the markers ( $\gamma \mathrm{GT}$, FFA, UCP2, PPAR $\delta$ and HSL).

In conclusion, ARO subjects had increased plasma levels of $\gamma \mathrm{GT}$ and FFA, and reduced PBMC gene expression level of UCP2, HSL and PPAR $\delta$ compared with MHO 
subjects. These metabolic differences may contribute to explain some of the underlying mechanisms responsible for the increased risk of disease among ARO subjects compared with MHO subjects. However, our results need to be confirmed in larger studies which also include clinical examination of liver fat content. A better understanding of the metabolic phenotypes of obese subjects may have important implications for future therapeutics.

Acknowledgments We thank Ellen Raael at Oslo and Akershus University College of Applied Sciences, Norway, and Ellen Sagen at Research Institute for Internal Medicine, University of Oslo, Rikshospitalet, Oslo University Hospital, Norway. This study was supported by Oslo and Akershus University College of Applied Sciences, Norway, The Throne Holst Foundation for Nutrition Research, The Nordic Center of Excellence on Systems biology in controlled dietary interventions and cohort studies, SYSDIET (nr 070014), and the Research Council of Norway (P.O. Box 2700 St. Hanshaugen, N-0131 Oslo).

Conflict of interest Vibeke H. Telle-Hansen, Bente Halvorsen, Knut Tomas Dalen, Ingunn Narverud, Nima Wesseltoft-Rao, Stine M. Ulven and Kirsten Holven have no conflicts of interest, or any financial or personal interest. Linda Granlund is a researcher and nutrition manager at Mills DA, Norway.

\section{References}

Barish GD, Narkar VA, Evans RM (2006) PPAR delta: a dagger in the heart of the metabolic syndrome. J Clin Invest 116:590-597. doi:10.1172/JCI27955

Bouwens M, Afman LA, Muller M (2007) Fasting induces changes in peripheral blood mononuclear cell gene expression profiles related to increases in fatty acid beta-oxidation: functional role of peroxisome proliferator activated receptor alpha in human peripheral blood mononuclear cells. Am J Clin Nutr 86:15151523

Bouwens M, Afman LA, Muller M (2008) Activation of peroxisome proliferator-activated receptor alpha in human peripheral blood mononuclear cells reveals an individual gene expression profile response. BMC Genom 9:262. doi:10.1186/1471-2164-9-262

Bouwens M, Grootte Bromhaar M, Jansen J, Muller M, Afman LA (2010) Postprandial dietary lipid-specific effects on human peripheral blood mononuclear cell gene expression profiles. Am J Clin Nutr 91:208-217. doi:10.3945/ajen.2009.28586

Brochu M, Tchernof A, Dionne IJ, Sites CK, Eltabbakh GH, Sims EA, Poehlman ET (2001) What are the physical characteristics associated with a normal metabolic profile despite a high level of obesity in postmenopausal women? J Clin Endocrinol Metab 86:1020-1025

Dalgaard LT (2011) Genetic variance in uncoupling protein 2 in relation to obesity, Type 2 diabetes, and related metabolic traits: focus on the functional $-866 \mathrm{G}>\mathrm{A}$ promoter variant (rs659366). J Obes 2011:340241. doi:10.1155/2011/340241

Dalgaard LT, Pedersen O (2001) Uncoupling proteins: functional characteristics and role in the pathogenesis of obesity and Type II diabetes. Diabetologia 44:946-965. doi:10.1007/s001250100596

Fisler JS, Warden CH (2006) Uncoupling proteins, dietary fat and the metabolic syndrome. Nutr Metab (Lond) 3:38. doi:10.1186/ 1743-7075-3-38
Hotamisligil GS (2006) Inflammation and metabolic disorders. Nature 444:860-867. doi:10.1038/nature05485

Karelis AD, Brochu M, Rabasa-Lhoret R (2004a) Can we identify metabolically healthy but obese individuals (MHO)? Diabetes Metab 30:569-572

Karelis AD, St-Pierre DH, Conus F, Rabasa-Lhoret R, Poehlman ET (2004b) Metabolic and body composition factors in subgroups of obesity: what do we know? J Clin Endocrinol Metab 89:25692575. doi:10.1210/jc.2004-0165

Livak KJ, Schmittgen TD (2001) Analysis of relative gene expression data using real-time quantitative PCR and the 2(-Delta Delta C(T)) method. Methods 25:402-408. doi:10.1006/meth.2001.1262

Marx N, Mackman N, Schonbeck U, Yilmaz N, Hombach V, Libby P, Plutzky J (2001) PPARalpha activators inhibit tissue factor expression and activity in human monocytes. Circulation 103: 213-219

Marx N, Kehrle B, Kohlhammer K, Grub M, Koenig W, Hombach V, Plutzky J (2002) PPAR activators as antiinflammatory mediators in human $\mathrm{T}$ lymphocytes: implications for atherosclerosis and transplantation-associated arteriosclerosis. Circ Res 90:703-710

Mendez-Sanchez N, Arrese M, Zamora-Valdes D, Uribe M (2007) Current concepts in the pathogenesis of nonalcoholic fatty liver disease. Liver Int 27:423-433. doi:10.1111/j.1478-3231.2007. 01483. $\mathrm{x}$

Mojiminiyi OA, Abdella NA, Al Mohammedi H (2010) Higher levels of alanine aminotransferase within the reference range predict unhealthy metabolic phenotypes of obesity in normoglycemic first-degree relatives of patients with type 2 diabetes mellitus. J Clin Hypertens (Greenwich) 12:301-308. doi:10.1111/j.17517176.2009.00238.x

Mulder H, Sorhede-Winzell M, Contreras JA, Fex M, Strom K, Ploug T, Holm C (2003) Hormone-sensitive lipase null mice exhibit signs of impaired insulin sensitivity whereas insulin secretion is intact. J Biol Chem 278:36380-36388. doi:10.1074/jbc.M2130 32200

Ooi EM, Watts GF, Sprecher DL, Chan DC, Barrett PH (2011) Mechanism of action of a peroxisome proliferator-activated receptor (PPAR)-delta agonist on lipoprotein metabolism in dyslipidemic subjects with central obesity. J Clin Endocrinol Metab 96:E1568-E1576. doi:10.1210/jc.2011-1131

Pecqueur C, Alves-Guerra C, Ricquier D, Bouillaud F (2009) UCP2, a metabolic sensor coupling glucose oxidatin to mitochondrial metabolism? IUBMB Life 61:762-767. doi:10.1002/iub.188

Reid BN, Ables GP, Otlivanchik OA, Schoiswohl G, Zechner R, Blaner WS, Huang LS (2008) Hepatic overexpression of hormone-sensitive lipase and adipose triglyceride lipase promotes fatty acid oxidation, stimulates direct release of free fatty acids, and ameliorates steatosis. J Biol Chem 283:13087-13099. doi: $10.1074 /$ jbc.M800533200

Riserus U, Sprecher D, Johnson T et al (2008) Activation of peroxisome proliferator activated receptor (PPAR)delta promotes reversal of multiple metabolic abnormalities, reduces oxidative stress, and increases fatty acid oxidation in moderately obese men. Diabetes 57:332-339

Sanderson LM, Degenhardt T, Koppen A, Kalkhoven E, Desvergne B, Muller M, Kersten S (2009) Peroxisome proliferator-activated receptor beta/delta (PPARbeta/delta) but not PPARalpha serves as a plasma free fatty acid sensor in liver. Mol Cell Biol 29:6257-6267. doi:10.1128/MCB.00370-09

Sekiya M, Osuga J, Yahagi N, Okazaki H, Tamura Y, Igarashi M, Ishibashi S (2008) Hormone-sensitive lipase is involved in hepatic cholesteryl ester hydrolysis. J Lipid Res 49:1829-1838. doi:10.1194/jlr.M800198-JLR200

Sims EA (2001) Are there persons who are obese, but metabolically healthy? Metabolism 50:1499-1504. doi:10.1053/meta.2001. 27213 
Strable MS, Ntambi JM (2010) Genetic control of de novo lipogenesis: role in diet-induced obesity. Crit Rev Biochem Mol Biol 45:199-214. doi:10.3109/10409231003667500

Taskinen MR, Adiels M, Westerbacka J, Söderlund S, Kahri J, Lundbom N, Lundbom J, Hakkarainen A, Olofsson SO, Orho-Melander M, Borén J (2011) Dual metabolic defects are required to produce hypertriglyceridemia in obese subjects. Arterioscler Thromb Vasc Biol 31:2144-2150

Vozarova B, Stefan N, Lindsay RS, Saremi A, Pratley RE, Bogardus C, Tataranni PA (2002) High alanine aminotransferase is associated with decreased hepatic insulin sensitivity and predicts the development of type 2 diabetes. Diabetes 51:1889-1895

Wannamethee SG, Shaper AG, Lennon L, Whincup PH (2005)

Hepatic enzymes, the metabolic syndrome, and the risk of type 2 diabetes in older men. Diabetes Care 28:2913-2918

Wildman RP (2009) Healthy obesity. Curr Opin Clin Nutr Metab Care 12:438-443. doi:10.1097/MCO.0b013e32832c6db7 